\title{
Effects of GPER-1 receptor activation on the reactivity of pulmonary vascular bed and its possible protective role on ischemia /reperfusion
}

\author{
Gökçen TELLİ 1 , Banu Cahide TEL 1, Kansu BÜYÜKAFSAR 2, Bülent GÜMÜŞEL ${ }^{1}$ * \\ 1 Department of Pharmacology, Faculty of Pharmacy, Hacettepe University, Ankara, Turkey. \\ 2 Department of Pharmacology, Faculty of Medicine, Mersin University, Mersin, Turkey. \\ * Corresponding Author. E-mail: gumusel@hacettepe.edu.tr (B.G.); Tel. +90-312-305 21 31; ORCID No: 0000-0002- \\ 7533-7949.
}

Received: 27 November 2017 / Revised: 26 January 2018 / Accepted: 28 January 2018

\begin{abstract}
Estrogens have remarkable roles in various diseases and physiological events. The recent studies indicate that estrogens exhibit rapid effects such as calcium influx or release of nitric oxide via receptors on the cell membrane or on endoplasmic reticulum. This receptor is called GPER-1 (G protein-coupled estrogen receptor-1). In many previous studies, acute administration of GPER-1 selective agonist G1 reduced ischemia/reperfusion (I/R) injury in heart. In this study, the effect of G1 on the pulmonary vascular bed was investigated using isolated organ bath and isolated lung perfusion. The effect of pre-ischemic acute G1 treatment on lung I/R injury was assessed with post-I/R acetylcholine (ACh) or sodium nitroprusside (SNP) administration. Lungs wet/dry weight ratios were measured to determine the role of acute G1 administration on lung edema due to I/R injury. G1 was not cause a vasorelaxation in precontracted pulmonary arteries. The acute G1 pre-administration was increased the responses of ACh and SNP in I/R injury, however this increase was not statistically significant compared to control. There was no change on lung wet/dry weight ratio between the treatment and control groups. The vasorelaxation responses to ACh and SNP were not different between male and female rats. In our study, G1 caused a slight vasorelaxation in pulmonary vascular bed. There was no significant increase in the vasorelaxations with G1 treatment after I/R injury. Our results suggest that considering the presence of GPER-1 in lung tissue, GPER-1 should be evaluated in detail with further studies.
\end{abstract}

KEYWORDS: GPER-1; ischemia/reperfusion injury; pulmonary vascular bed; G1.

\section{INTRODUCTION}

Estrogens are steroid sex hormones that are important in development and maintenance of female reproductive system. Furthermore, estrogens also exhibits remarkable effects in both male and female cardiovascular, endocrine, central nervous, musculoskeletal and immune systems [1] Estrogens pass the cell membrane easily because of the hydrophobic structures and show their effects by interacting two nuclear receptors, known as ERa (estrogen receptor alpha) and ER $\beta$ (estrogen receptor beta) [2-4]. However, it is shown that estrogen agonist 17- $\beta$-estradiol show some effects only a few minutes after receptor activation [2]. Hence, it was considered that estrogens also interact with the membrane receptors. Subsequently, a receptor was found responsible for some rapid effects of estrogens such as calcium influx and releasing of nitric oxide [5-8]. This receptor was called G protein coupled estrogen receptor-1 (GPER-1) and located in cell membrane or endoplasmic reticulum. GPER-1 is found extensively throughout the body especially in cardiovascular, urinary, reproductive, immune nervous systems and bones [2, 3, 9-12]. The role of GPER-1 in the response of estrogen draws attention in cardiovascular system. GPER-1 selective agonist G1 causes vasodilation in mesentery, renal, carotid, coronary arteries and aorta [13-16]. Furthermore, acute G1 treatment reduced infarct size of heart and post-ischemic contraction dysfunction [17-20]. Many different studies that were performed in heart indicated the protective role of G1 against I/R injury [17, 18, 20]. The expression of GPER-1 receptors in lungs was shown with northern blot [4]. However, the role of G1 in lung I/R was not examined yet. It was considered that, if acute G1 therapy protected the lungs against I/R damage, GPER-1 receptor may be considered as a possible therapeutic target to prevent or reduce ischemic damage that may occur after life-

How to cite this article: Telli G, Tel BC, Büyükafşar K, Gümüşel B. Effects of GPER-1 receptor activation on the reactivity of pulmonary vascular bed and its possible protective role on ischemia /reperfusion injury. Marmara Pharm J. 2018; 22 (3): $422-428$. 
threatening surgical procedures such as pulmonary thromboembolectomy or lung transplantation. In this study, based on the effects of G1 in heart, we investigated the role of G1 in pulmonary vascular bed. The possible protective effect of acute G1 treatment in pulmonary I/R injury was also examined. Estrogen levels show difference between male and female, therefore the role of G1 was investigated both male and female rats.

\section{RESULTS}

\subsection{Effect of $\mathrm{G} 1$ in pulmonary vascular bed}

In the precontracted main pulmonary arteries (PAs), G1 $\left(10^{-7}-3 \times 10^{-6} \mathrm{M}\right)$ provided a slight vasorelaxation. However, this relaxation was not statistically significant when compared with the control group. The specific GPER-1 antagonist G15 partly inhibited the vasorelaxation (Figure 1). There was no change of the basal tension of the PAs with direct administration of G1 $\left(10^{-7}-3 \times 10^{-6} \mathrm{M}\right.$, data were not shown).

The bolus intraarterial (i.a.) administration of G1 reduced the pulmonary arterial pressure that was increased with U46619. However, this reduction was not different with control group. The response to G1 in the presence of G15 was similar with the response to G1 alone (Figure 2). The direct bolus i.a. administration of G1 did also not change the basal perfusion pressure of the lungs (data were not shown).

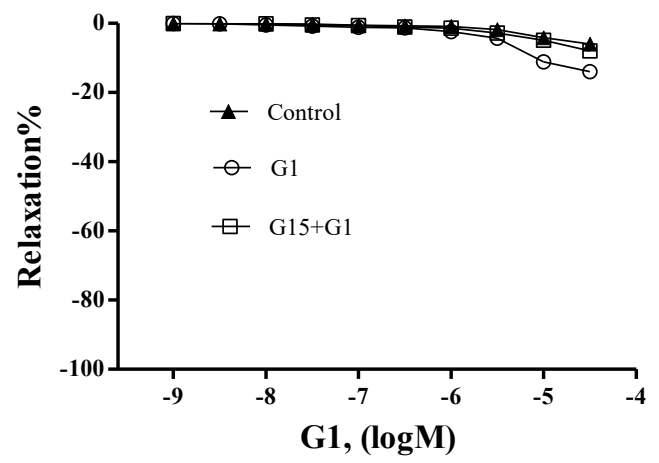

Figure 1. G1-induced vasorelaxation in the main PAs of rats. The vasorelaxation was not statistically significant compared to control group. The selective antagonist G15 was partly inhibited the vasorelaxation response of G1 and this inhibition also was not statistically significant. The statistical analysis were performed with Student's t test and data were expressed as mean \pm standart error mean (SEM), $\mathrm{n}=6-8$.

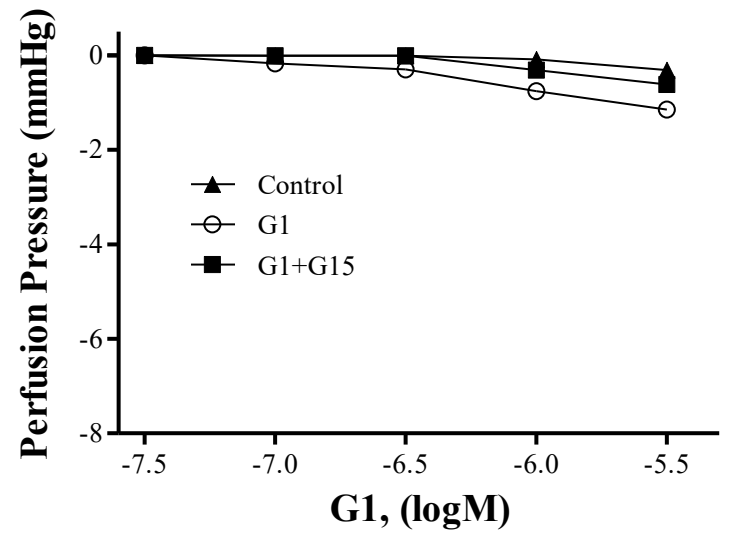

Figure 2. After i.a. G1 administration the reduction of the pulmonary arterial pressure was not statistically significant compared to control group. The selective antagonist G15 was partly inhibited the vasorelaxation response of G1 and this inhibition also was not statistically significant. The statistical analysis were performed with Student's $t$ test and data were expressed as mean \pm SEM, $n=6-8$. 


\subsection{Effect of acute G1 administration on vasorelaxation responses of PA subsequent ischemia/reperfusion injury}

I/R injury reduced the ACh-induced vasorelaxation compare to control group ( ${ }^{*} \mathrm{p}<0.05$; I/R group vs. control group). Preischemic acute administration of G1 provided a slight increase in the vasorelaxation response to ACh. However this increase did not reach statistical significance both male and female rats (Figure 3A and 3B).

A

Acetylcholine $(2 \mu \mathrm{g} / 100 \mu \mathrm{l})$

(Male Rats)

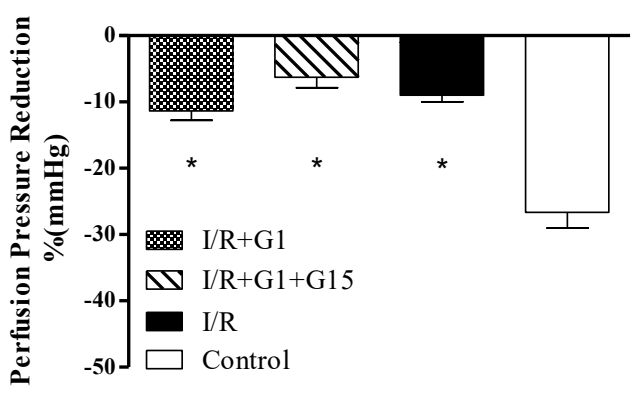

B

Acetylcholine $(2 \mu \mathrm{g} / 100 \mu \mathrm{l})$

(Female Rats)

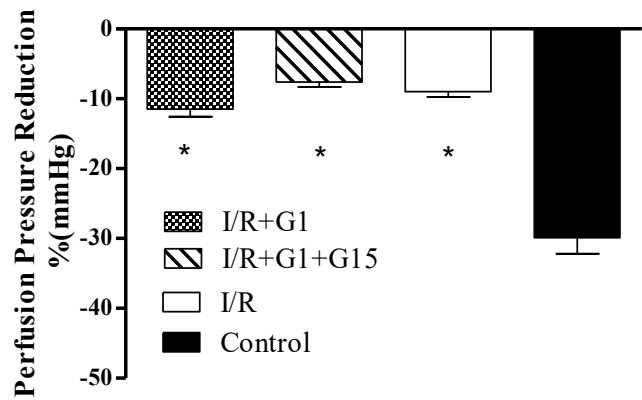

Figure 3. The vasorelaxation responses of acetylcholine in male rats isolated lungs. I/R injury reduced the acetylcholineinduced vasorelaxation. The acute G1 treatment did not reverse this reduction (A; * $\mathrm{p}<0.05 \mathrm{I} / \mathrm{R}$ groups vs control group). Similarly, in all I/R groups the vasorelaxation of acetylcholine was reduced in female rats $\left({ }^{*} \mathrm{p}<0.05 \mathrm{I} / \mathrm{R}\right.$ groups vs control group (B)), $\mathrm{n}=6-8$.

Similar with ACh, SNP-induced vasorelaxation reduced after I/R injury ( ${ }^{*} \mathrm{p}<0.05 \mathrm{I} / \mathrm{R}$ groups vs control group). The increase of SNP-induced vasorelaxation with acute G1 treatment was not statistically significant compared to I/R group (Figure $4 \mathrm{~A}$ and $4 \mathrm{~B}$ ).

The reduction in the perfusion pressure with administration of ACh or SNP were not different between male and female rats (Figure $5 \mathrm{~A}$ and $5 \mathrm{~B}$ ).

The wet/dry weight ratios of isolated lungs increased based on the I/R injury dependent-edema, ( ${ }^{*} \mathrm{p}<0.05 ; \mathrm{I} / \mathrm{R}$ group vs. control group). The G1 pretreatment did not reduce this increase (Figure 6A and 6B).

A

$\operatorname{SNP} 5 \mu \mathrm{g} / 100 \mu \mathrm{l}$

(Male Rats)

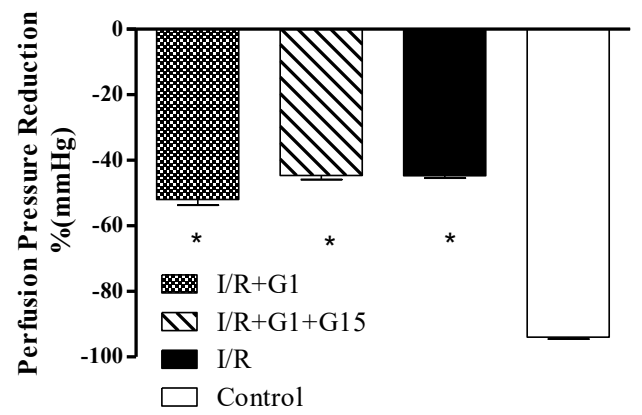

B

$\mathrm{SNP} 5 \mu \mathrm{g} / 100 \mu \mathrm{l}$

(Female Rats)

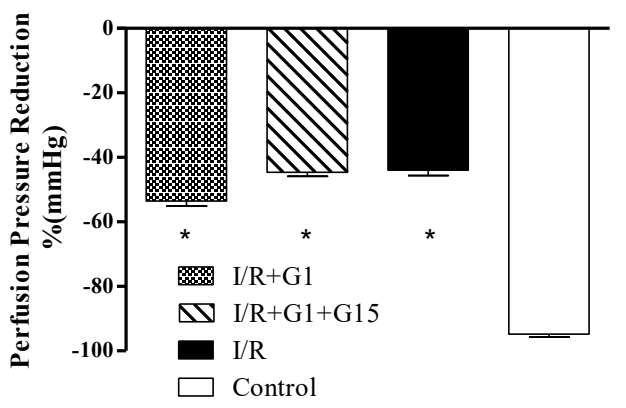

Figure 4. The vasorelaxation responses of SNP in male rats isolated lungs. I/R injury reduced the SNPinduced vasorelaxation. The acute G1 treatment did not reverse this reduction $\left({ }^{*} \mathrm{p}<0.05 \mathrm{I} / \mathrm{R}\right.$ groups vs control group). Similarly, in all I/R groups the vasorelaxation of SNP was reduced in female rats $\left({ }^{*} \mathrm{p}<0.05\right.$ I/R groups vs control group) $n=6$ for both groups. 
A

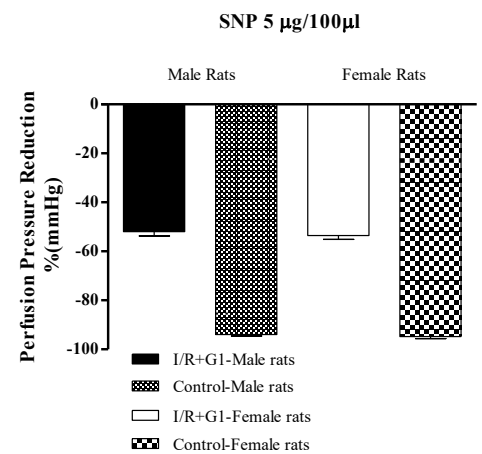

B

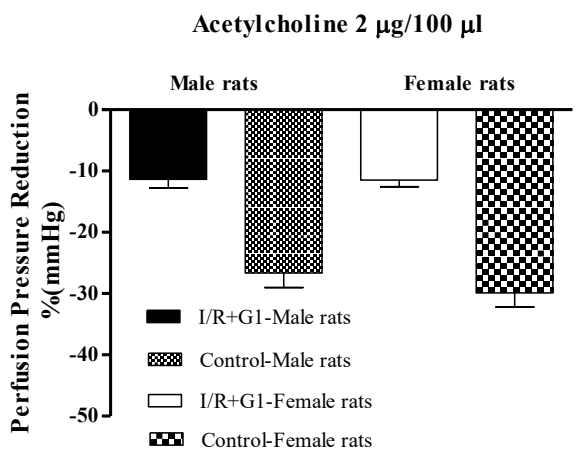

Figure 5. The vasorelaxation responses of acetylcholine and SNP were not different between male and female rats after G1 treatment in isolated lungs; $n=6$ for both groups. The wet/dry weight ratios of isolated lungs were increased based on the edema, which was to I/R injury dependent $\left({ }^{*} \mathrm{p}<0.05 ; \mathrm{I} / \mathrm{R}\right.$ group vs. control group). The G1 pretreatment did not reduce this increase (Figure 5A and 5B).

A

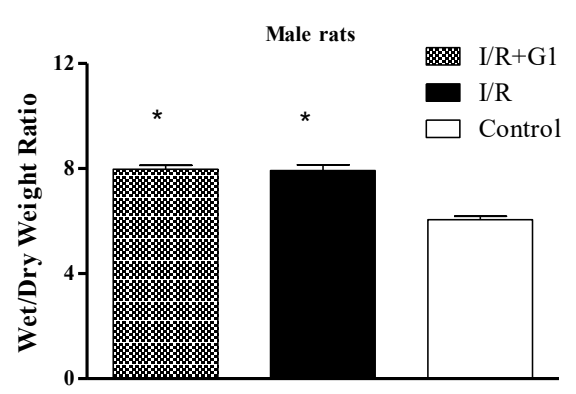

B

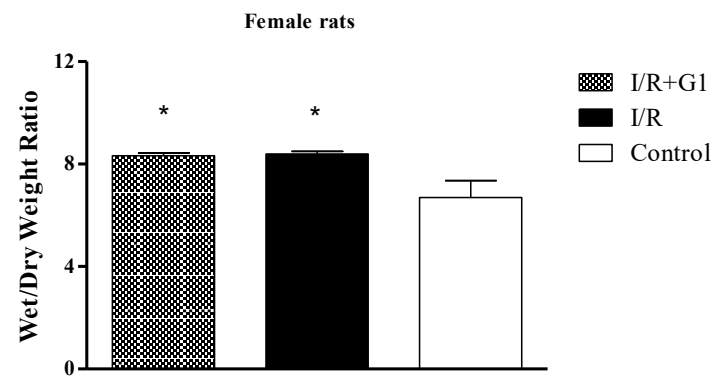

Figure 6. The wet/dry weight ratio of isolated lungs both male and female rats $\left({ }^{*} \mathrm{p}<0.05 ; \mathrm{I} / \mathrm{R}\right.$ group vs. control group, $\mathrm{n}=6$ for both groups).

\section{DISCUSSION}

In the present study the role of selective GPER-1 agonist G1 on pulmonary system was broadly investigated. The presence of the GPER-1 in lungs was demonstrated previously [4]. However, there are few studies investigating the effects of G1 in the pulmonary system. Reslan et al. showed that G1-induced vasorelaxation in female rat PA was not as potent as vasorelaxation of mesenteric, renal and carotid artery [16]. In our study, we also established that G1 provided a slight relaxation of male PAs. However, the vasorelaxation that was observed in the main PA was not statistically significant when compared to the control group. The direct administration of G1 did also not change the basal tension of the PAs. Therefore, we thought that G1 does not play an active role in vascular regulation in male main PAs. Isolated lung perfusion was performed to evaluate the role of G1 in the resistance arteries of pulmonary vascular bed. Initially the effect of G1 was examined on the lungs that perfusion pressure was increased with U46619. The reduction of the perfusion pressure with G1 administration was not statistically significant when compared to control group and was not fully inhibited in the presence of selective antagonist G15. There was also no effect of G1 on the basal perfusion pressure of the lungs. Hence, G1 did not seem a direct target for pulmonary vascular system diseases. However, it was demonstrated that prophylactic G1 treatment reduced the pulmonary hypertension $(\mathrm{PH})$-induced right ventricle dysfunction, it can be the results of G1-dependent releasing of nitric oxide [21, 22].

It is known that in post-menopausal women, the risk of cardiovascular diseases increases due to reduction in estrogens levels [23]. The role of GPER-1 in ischemic heart disease was investigated in many studies. Pre-ischemic acute G1 treatment reduced the I/R injury in both male and female rats [24]. Therefore we investigated the role of G1 in lung I/R injury. For this purpose, I/R injury was induced in isolated lungs from both female and male rats and the effect of acute G1 treatment on the I/R injury was assessed by ACh and SNP administration. Although there was an increase in relaxation responses between the control group and the pre-ischemic acute G1 treatment group, this increase was not statistically significant. When the 
wet/dry weight ratios of the lungs were evaluated, it was determined that acute G1 therapy did not reduce the amount of I/R injury-induced edema.

Although these results suggest that GPER-1 is not an important receptor in the pulmonary system, there are also many studies indicate the protective effect of GPER- 1 in cardiovascular system diseases. GPER-1 mediates the rapid effects of estrogens so it can be expected that selective agonist G1 might be effective in also pulmonary diseases with chronic treatment. Further studies should be perform to elucidate the role of the GPER-1 in the lung tissue. Considering the presence of GPER-1 in lung tissue, it still can be a potential target for therapy of pulmonary diseases.

\section{CONCLUSION}

In this study the role of GPER-1 in lung I/ R injury was investigated for the first time. The selective GPER-1 agonist G1 provided a slight vasorelaxation in pulmoner vascular bed and acute G1 treatment caused only a little improvement in vasorelaxation responses of ACh and SNP after I/R injury. Considering these promising results, the role of GPER-1 may investigate with further studies.

\section{MATERIALS AND METHODS}

\subsection{Animals}

Male (250-300g) and female (180-220g) Sprague Dawley (SD) rats were used in experiments. The rats were housed in a room with a $12 \mathrm{~h} / 12 \mathrm{~h}$ light/dark cycle at a constant temperature $\left(22 \pm 1^{\circ} \mathrm{C}\right)$, and were provided ad libitum access to food and water. All experiments were approved by the Hacettepe University Animal Experimentations Local Ethics Board (2013/21-A).

\subsection{Chemicals}

GPER-1 selective agonist G1 and selective antagonist G15 were purchased from Tocris (Bristol, United Kingdom). All other chemicals were purchased from Sigma Aldrich (St Louis, Missouri, USA). G1 and G15 were dissolved in DMSO, U46619 was dissolved in ethanol and all other chemicals were dissolved distilled water.

\subsection{Isolated Organ Bath}

Isolated organ bath experiments were performed in both male and female SD rats described as previously [25]. Briefly, the rats were euthanized by high-dose anesthesia (ketamine/xylazine) and lungs were isolated and immersed in cold Krebs-Henseleit solution (KHS, comprised of the following: $118 \mathrm{mM} \mathrm{NaCl}, 4.7$ $\mathrm{mM} \mathrm{KCl}, 2.5 \mathrm{mM} \mathrm{CaCl}_{2}, 1.2 \mathrm{mM} \mathrm{KH}_{2} \mathrm{PO}_{4}, 25 \mathrm{mM}, \mathrm{NaHCO}_{3}, 1.2 \mathrm{mM} \mathrm{Mg}_{2} \mathrm{SO}_{4}$, and $10 \mathrm{mM}$ glucose). Main PA was cleaned and cut into 2-3 mm-long rings. The PA rings were mounted in an organ bath containing $5 \mathrm{ml}$ of KHS. The solution was continuously gassed with $95 \%$ oxygen and $5 \% \mathrm{CO}_{2}$ at $37^{\circ} \mathrm{C}$. The isometric changes in tension were measured with a force transducer (MP36, Commat, Ankara, Turkey). The PAs were equilibrated for 60 minutes under a tension of $1.5 \mathrm{~g}$ by washing with KHS every 10 minutes. At the end of the resting period, the integrity of endothelium was determined with a single submaximal dose of $\left.\mathrm{ACh} ; 3 \times 10^{-6} \mathrm{M}\right)$. The endothelium was accepted intact when vasorelaxation were over 70\%. Initially, the direct effect of G1 on basal tonus of PA was assessed with cumulative administration $\left(10^{-9}-3 \times 10^{-5} \mathrm{M}\right)$. In another set of experiments, the PA rings were precontracted with U46619 $\left(3 \times 10^{-8} \mathrm{M}\right)$ at approximately $1 \mathrm{~g}$ and $\mathrm{G} 1\left(10^{-9}-3 \times 10^{-5} \mathrm{M}\right)$ was applied cumulatively. The response of G1 was repeated after the 30 minutes incubation of GPER-1 selective antagonist G15 $\left(3 \times 10^{-6} \mathrm{M}\right)$. In control group the vehicle (DMSO) was applied instead of selective agonist G1 and all procedure was repeated.

\subsection{Isolated Lung Perfusion}

Male and female SD rats were anesthesized with ketamine/xylazine $(90 / 10 \mathrm{mg} / \mathrm{kg})$. After tracheal cannulation the thoracic cavity was opened and heparin was injected into right ventricle. PA was cannulated and the animal was euthanized by exsanguination from the abdominal aorta. The main pieces of heart was cut, the heart-lung block was isolated. Lungs were mounted to the modified Langendorff system from PA perfusion cannula and perfused with KHS with a constant flow rate $(6 \mathrm{ml} / \mathrm{min})$ by using a peristaltic pump (Gilson Model M312, Middleton, USA). The changes in perfusion pressure was measured with a pressure transducer and recorded with an acquisition system (MP36, Commat, Ankara). Lungs were stabilized with 30 minutes and then direct effect of cumulative G1 $\left(10^{-7}-3 \times 10^{-6} \mathrm{M}\right)$ on perfusion pressure was assessed. The effect 


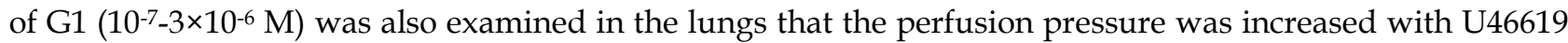
$\left(3 \times 10^{-8} \mathrm{M}\right)$. These experiments were repeated in the presence of G15 $\left(3 \times 10^{-6} \mathrm{M}\right)$. In control group the vehicle (DMSO) was applied instead of selective agonist G1 and all procedure was repeated.

\subsection{Ischemia/Reperfusion Model}

Male and female rats were used for the I/R experiments. The lungs were stabilized 20 minutes after mounted the modified Langendorff system. Then, G1 $\left(10^{-6} \mathrm{M}\right)$ was applied for 10 minutes. The lungs were removed in KHS solution at $4^{\circ} \mathrm{C}$ in refrigerator for 20 minutes (ischemia period). At the end of this period, lungs were reperfused for 60 minutes (reperfusion period) [24]. Another set of experiments, the lungs were pretreated with G15 $\left(3 \times 10^{-6} \mathrm{M}\right)$ for 20 minutes and G1 was administrated in the presence of G15 $\left(3 \times 10^{-6} \mathrm{M}\right)$. Then, I/R protocol was performed similarly. In the control group the vehicle ( $1 \% \mathrm{DMSO})$ was applied instead of G1. In all set of experiments the perfusion pressure was increased with U46619 $\left(3 \times 10^{-8} \mathrm{M}\right)$ and i.a. bolus ACh $(2 \mu \mathrm{g} / 0.1 \mathrm{ml})$ or SNP was applied. The lungs were weighed at the end of the experiments and then were dried in the oven at $80^{\circ} \mathrm{C}$ for 24 hours. The dried lungs were also weighed and the wet weight / dry weight ratio was calculated. In control group the vehicle (DMSO) was applied instead of selective agonist G1 and all procedure was repeated.

\subsection{Statistical Analysis}

All data were expressed as mean \pm SEM values. Statistical analysis was performed with two-way ANOVA in isolated organ bath experiments. The analysis of isolated lung perfusion and I/R injury were performed by one-way ANOVA. A p-value of less than 0.05 was considered statistically significant.

Acknowledgements: This study was supported by the Hacettepe University Scientific Research Projects Coordination Unit (Project numbers: 013 D08 301 002-350).

Author contributions: Concept - G.T., B.C.T., K.B., B.G.; Design - G.T., B.C.T., B.G.; Supervision - B.C.T., B.G.; Resource - G.T.; Materials - G.T.; Data Collection and/or Processing - G.T.; Analysis and/or Interpretation - G.T.; Literature Search - G.T. Writing - G.T.; B.C.T., K.B., B.G. Critical Reviews - G.T., B.C.T., K.B., B.G.

Conflict of interest statement: The authors declared no conflict of interest.

\section{REFERENCES}

[1] Heldring N(1), Pike A, Andersson S, Matthews J, Cheng G, Hartman J, Tujague M, Ström A, Treuter E, Warner M, Gustafsson JA. Estrogen receptors: How do they signal and what are their targets. Physiol Rev. 2007;87(3):905-931.

[2] Filardo EJ, Thomas P. Minireview: G protein-coupled estrogen receptor-1, GPER-1:Its mechanism of action and role in female reproductive cancer, renal and vascular physiology. Endocrinology. 2012;153(7):2953-2962.

[3] Nilsson BO, Olde B, Leeb-Lundberg LM. G protein-coupled oestrogen receptor 1 (GPER1)/GPR30: a new player in cardiovascular and metabolic oestrogenic signalling. Br J Pharmacol. 2011;163(6):1131-1139.

[4] Mizukami Y. In vivo functions of GPR30/GPER-1, a membrane receptor for estrogen: from discovery to functions in vivo. Endocr J. 2010;57(2):101-107.

[5] Razandi M, Pedram A, Greene GL, Levin ER. Cell membrane and nuclear estrogen receptors (ERs) originate from a single transcript: studies of ERalpha and ERbeta expressed in Chinese hamster ovary cells. Mol Endocrinol. 1999;13(2):307-319.

[6] Carmeci C, Thompson DA, Ring HZ, Francke U, Weigel RJ. Identification of a gene (GPR30) with homology to the G-protein-coupled receptor superfamily associated with estrogen receptor expression in breast cancer. Genomics. 1997;45(3):607-617.

[7] Filardo EJ, Quinn JA, Bland KI, Frackelton AR, Jr. Estrogen-induced activation of Erk-1 and Erk-2 requires the G protein-coupled receptor homolog, GPR30, and occurs via trans-activation of the epidermal growth factor receptor through release of HB-EGF. Mol Endocrinol. 2000;14(10):1649-1660.

[8] Filardo EJ, Quinn JA, Frackelton AR, Jr, Bland KI. Estrogen action via the G protein-coupled receptor, GPR30: stimulation of adenylyl cyclase and cAMP-mediated attenuation of the epidermal growth factor receptor-to-MAPK signaling axis. Mol Endocrinol. 2002;16(1):70-84.

[9] Edwards DP. Regulation of signal transduction pathways by estrogen and progesterone. Annu Rev Physiol. 2005;67:335-376. 
[10] Prossnitz ER, Barton M. The G-protein-coupled estrogen receptor GPER in health and disease. Nat Rev Endocrinol 2011;7(12):715-726

[11] Filardo EJ, Thomas P. GPR30: A seven-transmembrane-spanning estrogen receptor that triggers EGF release. Trends Endocrinol Metab. 2005;16(8):362-367.

[12] Prossnitz ER, Arterburn JB, Smith HO, Oprea TI, Sklar LA, Hathaway HJ. Estrogen signaling through the transmembrane G protein-coupled receptor GPR30. Annu Rev Physiol. 2008;70:165-190.

[13] Meyer MR, Baretella O, Prossnitz ER, Barton M. Dilation of epicardial coronary arteries by the G protein-coupled estrogen receptor agonists G-1 and ICI 182,780. Pharmacology. 2010;86(1):58-64.

[14] Lindsey SH, Cohen JA, Brosnihan KB, Gallagher PE, Chappell MC. Chronic treatment with the G protein-coupled receptor 30 agonist G-1 decreases blood pressure in ovariectomized mRen2.Lewis rats. Endocrinology. 2009;150(8):3753-3758.

[15] Broughton BR, Miller AA, Sobey CG. Endothelium-dependent relaxation by G protein-coupled receptor 30 agonists in rat carotid arteries. Am J Physiol Heart Circ Physiol. 2010;298(3):H1055-1061.

[16] Reslan OM, Yin Z, do Nascimento GR, Khalil RA. Subtype-specific estrogen receptor-mediated vasodilator activity in the cephalic, thoracic, and abdominal vasculature of female rat. J Cardiovasc Pharmacol. 2013;62(1):26-40.

[17] Deschamps AM, Murphy E, Sun J. Estrogen receptor activation and cardioprotection in ischemia reperfusion injury. Trends Cardiovasc Med. 2010;20(3):73-78.

[18] Bopassa JC, Eghbali M, Toro L, Stefani E. A novel estrogen receptor GPER inhibits mitochondria permeability transition pore opening and protects the heart against ischemia-reperfusion injury. Am J Physiol Heart Circ Physiol. 2010;298(1):H16-23.

[19] Patel VH, Chen J, Ramanjaneya M, Karteris E, Zachariades E, Thomas P, Been M, Randeva HS. G-protein coupled estrogen receptor 1 expression in rat and human heart: Protective role during ischaemic stress. Int J Mol Med. 2010;26(2):193-199.

[20] Weil BR, Manukyan MC, Herrmann JL, Wang Y, Abarbanell AM, Poynter JA, Meldrum DR. Signaling via GPR30 protects the myocardium from ischemia/reperfusion injury. Surgery. 2010;148(2):436-443.

[21] Alencar AK, Montes GC, Montagnoli T, Silva AM, Martinez ST, Fraga AG, Wang H, Groban L, Sudo RT, Zapata-Sudo G. Activation of GPER ameliorates experimental pulmonary hypertension in male rats. Eur J Pharm Sci. 2017;97:208217.

[22] Fredette NC, Meyer MR, Prossnitz ER. Role of GPER in estrogen-dependent nitric oxide formation and vasodilation. J Steroid Biochem Mol Biol. 2017; 176: 65-72.

[23] White RE. Estrogen and vascular function. Vascul Pharmacol. 2002;38(2):73-80.

[24] Deschamps AM, Murphy E. Activation of a novel estrogen receptor, GPER, is cardioprotective in male and female rats. Am J Physiol Heart Circ Physiol. 2009;297(5):H1806-813.

[25] Telli G, Tel BC, Yersal N, Korkusuz P, Gumusel B. Effect of intermedin/adrenomedullin2 on the pulmonary vascular bed in hypoxia-induced pulmonary hypertensive rats. Life Sci. 2017; 192: 62-67. 